\title{
Probing Gravitational Theories with Eccentric Eclipsing Detached Binary Stars
}

\author{
Leopoldo Milano ${ }^{1,2}$, Rosario De Rosa ${ }^{1,2}$, Mariafelicia De Laurentis ${ }^{1,2}$, Fabio Garufi ${ }^{1,2}$ \\ ${ }^{1}$ Dipartimento di Fisica Universita' Federico II di Napoli Complesso Universitario di Monte S. Angelo, Via Cinthia, - I \\ 80125, Napoli, Italy \\ ${ }^{2}$ Sezione INFN di Napoli, Complesso Universitario di Monte S. Angelo, Via - I 80125, Napoli, Italy
}

Corresponding author: milano@na.infn.it

\begin{abstract}
In this paper, we compare the effects of different theories of gravitation on the apsidal motion of eccentric eclipsing detached binary stars. The comparison is performed by using the formalism of the post-Newtonian parametrization to calculate the theoretical advance at periastron and compare it to the observed one, after having considered the effects of the structure and rotation of the involved stars. A variance analysis on the results of this comparison shows that no significant difference can be found due to the effect of the different theories under test with respect to the standard general relativity (GR). It will be possible to observe differences, as we would expect, by checking the observed period variation on a much larger lapse of time.
\end{abstract}

Keywords: relativity - gravitation - eclipsing binaries - apsidal motion.

\section{Introduction}

The problem of the motion of two bodies under their mutual gravitational attraction and the study of binary stellar systems has always been the ideal test bed for the theories of gravitation. Several authors in the last decades dedicated a lot of work in analyzing, both on the theoretical and the experimental point of wiew, the phenomenon of the periastron precession in binary systems to test various gravitational theories [1, 2] as well as to find correction to the Newtonian and General Relativistic behaviour of the systems due to stellar form factors, spin, tides and other phenomena [3].

The classical effect of General Relativity (GR) on the apsidal motion rate at periastron is well known since long time and described by Levi-Civita in a famous paper in 1937 [4, 5]. Another possible formulation of the problem, that allows also to test other gravitational theories besides GR, is the use of Parametrized Post Newtonian (PPN) formalism [6, 7]. Using this formalism, the different gravitational theories can be compared side by side on the basis of a set of Post-Newtonian (PN) parameters: the masses, the system major semiaxis and the eccentricity. Thus, using a sample of Eccentric Eclipsing Detached Binary (EEDB) systems, for which masses and orbital parameters are known with sufficient precision, it is possible to compare the apsidal motion rate at periastron $\dot{\omega}_{T h}$, as expected in the different theories with the observations in order to verify whether the observations can select one theory or another.

The choice of this class of stellar objects to test the theories is dictated by the circumstance that the both orbital and the structural parameters can be precisely determined by observation of the eclypses (the only alea being the orbit plane observation angle), and that in close stellar orbits, the gravitational field is supposed to be strong, thus enhancing eventual effects of deviations from the classical theory.

There are so many aspects of binary star evolution and angular momentum exchange (see, e.g., [8]) that any attempt to dig out from these other effects the subtleties of GR could seem futile, nonetheless, we consider useful to ascertain whether from the observation of many binary systems, some statistical information about the prevalence of one or another theory of gravitation can be extracted and to ground the bases of a method that can be used when other more significant data will be available.

\section{Advance at Periastron}

The idea of considering relativistic gravitational tests in terms of a metric expansion is originally based on a work by Shiff [9] who expanded the single body metric in terms of the ratio between the geometrized mass $m_{g}=G m / c^{2}$ and the distance $r$ :

$$
g_{00}=1-2 \alpha \frac{m_{g}}{r}+2 \beta\left(\frac{m_{g}}{r}\right)^{2}
$$




$$
\begin{gathered}
g_{0 k}=0 \\
g_{i k}=-\left(1+2 \gamma \frac{m_{g}}{r}\right) \delta_{i k} \quad i, k=1,2,3
\end{gathered}
$$

Four new parameters $\alpha^{\prime}, \alpha^{\prime \prime}, \alpha^{\prime \prime \prime}, \Delta$ were then introduced to account for relative velocities and accelerations. For General Relativity all the parameters are equal to 1 and the advance at periastron can be verified to reproduce the "classical" formula by Levi Civita [4]. We conveniently modified the 'classical' formula by introducing a factor $\left(K_{T h}\right)$ to take into account the dependance on the theory dependent PN terms in order to test the different relativistic theories. [10]:

$$
\dot{\omega}_{R e l}=1.8167 \times 10^{-4} K_{T h} \frac{\left(\frac{M}{P}\right)^{\frac{2}{3}}}{c^{2}\left(1-e^{2}\right)}
$$

where:

$$
K_{T h}=\frac{\alpha_{T h}\left(8 \Delta_{T h}-\alpha_{T h}+2 \alpha_{T h}^{\prime \prime}-\alpha_{T h}^{\prime \prime \prime}-\gamma_{T h}-\alpha_{T h}^{\prime}\right)}{2}
$$

The PN parameters, are calculated for the different gravitational theories, i.e. the General Relativity ('classical' term), the Nordvedt, the Brans-Dicke theories and the so called $f(R)$ theories that take into account higher order terms of the Ricci scalar $R$, giving a general expression for the relativistic term $\dot{\omega}_{R e l}$, that contributes to the advance at periastron. Using for the different theories the appropriate values of $\alpha_{T h}, \alpha_{T h}^{\prime}, \alpha_{T h}^{\prime \prime}, \alpha_{T h}^{\prime \prime \prime}, \Delta_{T h}$ and $\gamma_{T h}$ the numerical values of $K_{T h}$ can be obtained [10]:

$$
K_{T h}=\left\{\begin{array}{l}
K_{G R}=3 \rightarrow(\text { General }- \text { Relativity }) \\
K_{B D}=\frac{19}{7} \rightarrow(\text { Brans }- \text { Dicke }) \\
K_{N D}=\frac{11}{4} \rightarrow(\text { Nordvedt }) \\
K_{f(R)}=\frac{13}{4} \rightarrow(f(R))
\end{array}\right.
$$

\section{Comparison with Experimental Data}

To test the effects of deviations from the GR, we choose to study binary stellar systems with small radius orbits, so that the gravitational field is strong enough to evidence these deviations, if any. Among the various binary stars catalogues available in literature, we choose a sample of Eccentric Eclipsing Detached Binary stars such that the period, the eccentricity, the masses of the components, and, possibly, the observed internal structure function are known with a good precision as e.g. [11].

For these systems the passage at periastron precedes in a way that is precisely predictable from the gravitational theory, once given the stellar parameters such as masses, radius of the components, and orbital parameters.
To compare the global rate of theoretical apsidal motion in a binary system with the measured one we must take into account the individual contributions of each component due to tidal and rotational distortions, and the general relativistic term $\dot{\omega}_{\mathrm{Th}}$, where the index $T h$ indicates the theory under test (e.g. $\omega_{G R}$ for General Relativity). Assuming that rotation of both components of an eclipsing binary system is perpendicular to the orbital plane, the apsidal motion rate, $\dot{\omega}$ is given by the following simple relation [12]:

$$
\dot{\omega}_{O b s}=\dot{\omega}_{c l}+\dot{\omega}_{R e l}
$$

Where $\dot{\omega}_{c l}$ is the classical Newtonian term and $\dot{\omega}_{R e l}$ is the relativistic contribution of Eq. 2 and Eq. 4. The dependance of $\dot{\omega}_{c l}$ on the binary system parameters is expressed through the Internal Second-order Constants (ISC):

$$
\begin{aligned}
\bar{k}_{2 T h} & =\frac{c_{21} k_{21 T h}+c_{22} k_{22 T h}}{c_{21}+c_{22}} \\
\bar{k}_{2 O b s} & =\frac{\dot{\omega}_{c l}}{c_{21}+c_{22}}
\end{aligned}
$$

where the parameters $c_{2 i}$ are related to the masses and the orbital eccentricity of the binary system. It must be noticed that the individual ISC's $k_{2, i}$ cannot be obtained from the observations although they can be interpolated from evolutionary codes like those used in $[13,14]$.

So we can evaluate a mean model dependent $\bar{k}_{2 T h}$ and a mean observation dependent $\bar{k}_{2 O b s}$, and compare them to test the evolution stellar models from the observations of apsidal motion. Taking into account that $\bar{k}_{2 O b s}$ is generally smaller than $\bar{k}_{2 T h}$ (this means that the evolution models predict stellar cores less dense than those found by observed data), remembering Eq. 2 and Eq. 5 we see that $\dot{\omega}_{c l}$ will vary according to $\dot{\omega}_{T h}$. In this way we can test the different relativistic theories of gravitation by verifying whether the agreement of the model dependent mean ISC, with those coming from observations is significantly improved by using the different $\dot{\omega}_{T h}$ relativistic terms.

In Fig. 1 we show the trend of the apsidal motion rate $\dot{\omega}_{G R}$ vs $\dot{\omega}_{G R, B D, N D, f(R)}$. It results: $\dot{\omega}_{B D} \cong$ $0.92 \dot{\omega}_{G R}, \dot{\omega}_{N D} \cong 0.90 \dot{\omega}_{G R}, \dot{\omega}_{f(R)} \cong 1.10 \dot{\omega}_{G R}$. Obviously the numerical coefficients are the ratios $\frac{K_{T h}}{K_{G R}}$ (see Eq. 4). It is interesting to note that the $f(R)$ theory gives a relativistic contribution that is slightly higher than $G R, B D$ and $N D$. Moreover, $G R$ is $\approx$ the mean between $f(R)$ and $\mathrm{BD}$ and ND theories. It is also evident that there is no significant difference among the theories under test within the errors. In Fig. 2 we show the trend of observed $\dot{\omega}_{O b s}$ vs $\dot{\omega}_{R e l}$ for different relativistic terms $(T h \equiv G R, B D, N D, f(R))$. The red line is the trend of $\dot{\omega}_{R e l}=\dot{\omega}_{O b s}$. It is evident that, apart 
from a few systems, the relativistic term is always less than the observed one.

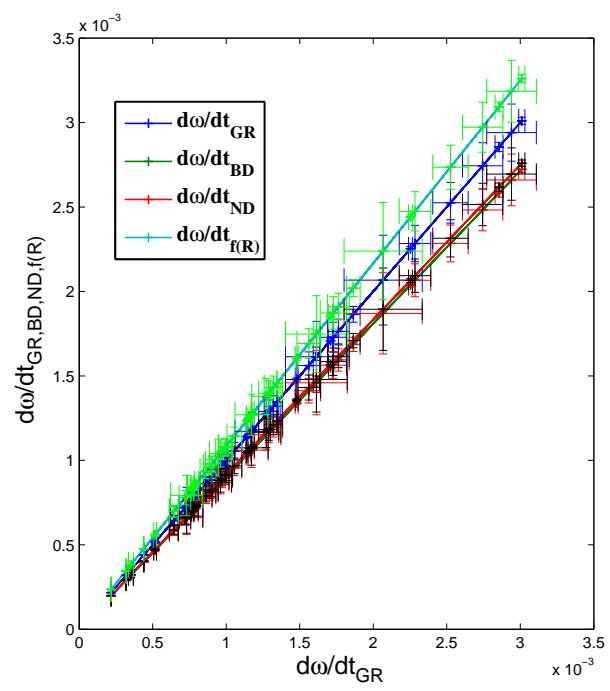

Figure 1: $\dot{\omega}_{G R}$ vs $\dot{\omega}_{G R, B D, N D, f(R)}$ for the different relativistic theories $(\mathrm{GR}, \mathrm{BD}, \mathrm{ND}, \mathrm{f}(\mathrm{R})): \dot{\omega}_{G R}=\dot{\omega}_{G R}$ $\dot{\omega}_{B D} \cong 0.92 \dot{\omega}_{G R}, \dot{\omega}_{N D} \cong 0.90 \dot{\omega}_{G R}, \dot{\omega}_{f(R)} \cong 1.10 \dot{\omega}_{G R}$

Fig. 3 shows the ISC's comparison: $\log \left(\bar{k}_{2 O b s_{T h}}\right)$ vs $\log \left(\bar{k}_{2 \text { StellarModel }}\right)$ are shown for different relativistic terms. The blue line is the trend $\mathrm{o}$ : $\log \left(\bar{k}_{2 \text { StellarModel }}\right)=\log \left(\bar{k}_{2 O s_{T h}}\right)$. It is evident that apart from a few systems, $\log \left(\bar{k}_{2 \text { StellarModel }}\right)$ is always greater than the observed one. So the stellar core densities derived from the observations is higher than those coming from stellar model prevision.

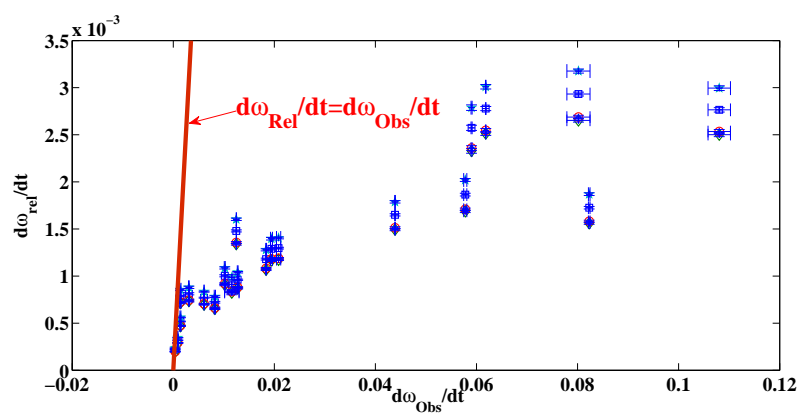

Figure 2: Observed $\dot{\omega}_{O b s}$ vs $\dot{\omega}_{R e l}$ for different relativistic terms $(T h \equiv G R, B D, N D, f(R))$. The red line is the trend of $\dot{\omega}_{R e l}=\dot{\omega}_{O b s}$. It is evident that apart from some systems the relativistic term is always less than the observed one.

\section{Discussion and Conclusion}

Using data coming from apsidal motion rate of EEDB we compared the variation of the relativistic term of the apsidal motion rate due to different theories of gravitation, that accordingly produces variation of classical Newtonian term (see Eq. 5). The results of this comparison was that we could not find any significant difference due to the effect of the different theories under test with respect to the standard General Relativity. Since the advance at periastron accumulates, the trend and the amount of this motion can be better determined by the observation of more orbits (or a larger fraction of orbit). Longer observations also improve the determination of ISC's; thus, it would be possible, perhaps, to observe more significant differences, by checking the period variation on a much larger lapse of time and verifying the assumptions of syncronous orbital and rotation motion of the binary star components. A lot of observing work are producing new and more accurate data and step by step it is getting a better agreement between theory and observations.

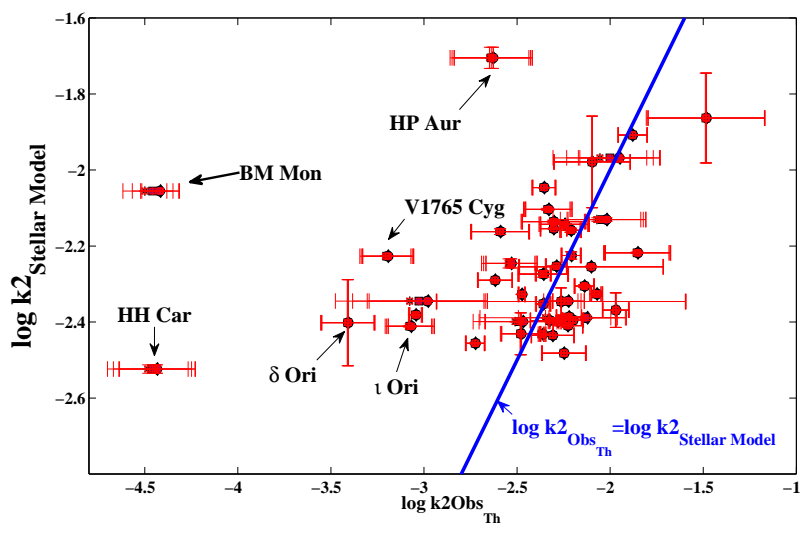

Figure 3: Internal second order structure constants (ISC) $\log \left(\bar{k}_{2 \mathrm{Obs}_{T h}}\right)$ vs $\log \left(\bar{k}_{2 \text { StellarModel }}\right)$ for different relativistic terms $(T h \equiv G R, B D, N D, f(R)$ ). The blue line is the trend of $\log \left(\bar{k}_{2 \text { StellarModel }}\right)=$ $\log \left(\bar{k}_{2 O b s_{T h}}\right)$. It is evident that apart from some systems $\log \left(\bar{k}_{2 \text { StellarModel }}\right)$ is always greater than the observed one. So the stellar core according to the observations is more dense than the stellar model prevision.

\section{Acknowledgement}

This work was supported by INFN grant 2011. The authors gratefully acknowledge the referee Prof. Todor Stanev for his useful suggestions.

\section{References}

[1] Breen B., 1973, J. Phys. A: Math., Nucl. Gen., 6, 150. 
[2] L. Lin-Sen, 2010, Astrophys. Space Sci., 327, 59. doi:10.1007/s10509-010-0267-4

[3] Giménez A., Claret A., 2010, Astron. and Astroph. 519, A57.

[4] Levi Civita T., 1937, Amer. J. of Math., 59, 225. doi:10.2307/2371404

[5] Giménez A., 1985, Astroph. J., 297, 405. doi:10.1086/163539

[6] Thorne K.S., Will C.M., 1971, Astrophys. J., 163, 595. doi:10.1086/150803

[7] Nordtvedt K., 1969, Phys. Rev., 180, 1293. doi:10.1103/PhysRev. 180.1293

[8] Biermann, P., et al. 1985, Astrophys. J., 293, 303

[9] Schiff L.I. , 1967, Relativity Theory and Astrophysics vol 1, ed J Ehlers 1967 (Philadelphia: American Mathematical Society)

[10] M. De Laurentis,R. De Rosa,F. Garufi \& L. Milano,2012,MNRAS, 424, 2371-2379

[11] Bulut I., Demircan O., 2007, Mon. Not. R. Astron. Soc. $378,179$.
[12] Kopal Z., Dynamics of Close Binary Systems. Reidel,Dordrecht (1978)

[13] Claret A., Giménez A., 1992, Astron. and Astroph. Suppl. 96, 255.

[14] Claret, A. 2004, A\&A, 424, 919 doi:10.1111/j.1365-2966.2007.11756.x

\section{DISCUSSION}

JIM BEALL: What systems would give a proper test?

LEOPOLDO MILANO: The systems that give a proper test are about eleven.

CARLOTTA PITTORI: Do you think that it could be useful to study in more datail the outliers? Which kind of obsevations could help to constrain the theory?

LEOPOLDO MILANO: Many group of astronomers are doing new observations on the outliers with the aim of improving the knowledge on the rotations velocities and other parameters that can be the cause of the failure of the simple model that is generally adopted. 